\title{
An Ice Core Slice Scanning Tool based on Line-scan Camera
}

\author{
Xiao Jiang ${ }^{1}$, Qi Yang ${ }^{2}$, Jianguang $\mathrm{Shi}^{2}$, Jingbiao Liu ${ }^{1}$, Haibin $\mathrm{Yu}^{2 *}$, and Chunlei $\mathrm{An}^{3 *}$ \\ ${ }^{1}$ Ocean Technology and Equipment Center, Hangzhou Dianzi University, Hangzhou, Zhejiang, 310018, China \\ ${ }^{2}$ College of Electronics and Information, Hangzhou Dianzi University, Hangzhou, Zhejiang, 310018, China \\ ${ }^{3}$ Polar Research Institute of China, Shanghai, 200136, China
}

\begin{abstract}
The ice core from polar ice sheet is one of the most valuable archives of past climate and environment. The ice core visual stratigraphy, based on optical scanning analysis, provides the most intuitive information of the micro-particles and/or air bubbles in the ice. At present, only few labs can perform high quality visual stratigraphy analysis in the world. Here, we develop an ice core slice scanning tool based on line-scan camera. The machine uses the high-resolution characteristics of the line-scan camera to complete high-resolution imaging of the ice core slices through linkage with the linear light sources. A motor drive and control system is developed to complete the uniform scanning control of the line-scan camera and light sources. A special control program and human-computer interaction interface are developed to realize the parameter setting, motor control and imaging result display. The test results show that the proposed ice core slice scanning tool meets the design requirements and its imaging results are significantly better than areascan cameras under the same conditions.
\end{abstract}

\section{Introduction}

In polar regions, e.g., Antarctica and Greenland, there are ice sheets with thickness of thousands of meters. The information of past climate and environment could be preserved in the ice. Since the polar regions are far away from human activities, the preserved information is rarely diffused, which makes the ice cores from these regions the most valuable archives of past climate and environment [1].

The characteristic of the micro-particles and air bubbles in the ice may change with its depth (depositional environment), making the scattered light intensity of ice different. Imaging analysis on the ice cores can acquire important optical characteristics such as bubble density and particles distribution. Meanwhile, the ice core is in a transparent or translucent state because it is solid water, which is very conducive to the introduction of auxiliary light sources to improve imaging quality. Therefore, optical scanning analysis of ice core slices has become a very important method for ice core research [2].

At present, most existing ice core optical scanning and analysis systems are based on the combination of traditional area-scan cameras and auxiliary light sources. For example, Takata $M$ et al. [3] used different light sources, such as high-frequency fluorescent lamps, lasers and LEDs, to construct an ice core optical scanner with a movable area-scan CCD camera. Bjorn S et al. [4] developed an ice core image analyzer for firn density analysis consisting of a movable $500 \mathrm{~W}$ pixel area-scan camera and a fixed side light source. Christophe $\mathrm{K}$ et al.
[5] used an area-scan camera to perform overlapping ice core imaging in a narrow band-shaped black box and three fixed positions to complete the ice core density and snow melting rate analysis.

Considering the continuous in-depth research on the optical characteristics of the ice cores, the resolution of the traditional area-scan cameras can no longer meet the research needs. Compared with the area-scan camera, the photosensitive devices of a line-scan camera are uniformly arranged in a row and imaged in one-dimensional mode. When it is matched with a uniform motion mechanism, extremely high imaging resolution can be achieved. Therefore, some researchers have applied it to the ice core optical scanning imaging [6-8]. But in China, no researchers have used line-scan cameras for ice core optical scanning. Although the line-scan camera has extremely high resolution, it requires a long exposure time, so that it cannot shoot moving objects, nor can it perform flash photography. It requires a fixed brightness light source to work closely with it.

For visual stratigraphy of the ice core, this study refers to the tool structure proposed by Anja K et al. [6], and independently proposes a high-resolution ice core slice scanning tool based on the line-scan camera. The main contributions of this study are as follows,

(1) A simple ice core slice scanning tool structure is designed, which uses a linear light source to cooperate with a line-scan camera to complete the high-resolution imaging and analysis of the ice core slice.

(2) For the linear scanning requirements of the ice core slice, a motor drive and control system is developed to complete the uniform scanning control of the line-scan camera and the linear light source.

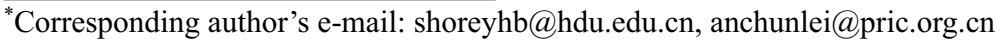


(3) A special man-machine interface is developed to complete parameter setting, scanning imaging, image display and analysis.

The remainder of this study is organized as follows. The structure of the proposed tool is given in Section 2. Section 3 describes the implementation of the proposed tool in detail. Section 4 presents the system test results. Finally, Section 5 concludes the study.

\section{Structure of the proposed ice core slice scanning tool}

The overall structure of the proposed ice core slice scanning tool based on the line-scan camera is shown in Figure 1. The tool mainly includes six parts: (1) An ice core imaging system composed of a line-scan camera, lens and capture card, which is responsible for obtaining highresolution images of the ice core slice and transmitting them to the computer. (2) An illumination system composed of two linear light sources, which keeps a certain angle and obliquely illuminates the ice core slice, so that a striped bright spot will be formed at the position facing the imaging plane of the camera. At the same time, the illumination system moves with the line-scan camera with the cooperation of the fixed axis, so that the striped bright spot also moves synchronously with the imaging plane of the camera. (3) A camera motion control system composed of a guide rail, a stepper motor and a motor controller, which ensures that the camera moves at a constant speed along the rail to complete the scanning and imaging of ice core slices. (4) A temperature control system, which controls the heating device to maintain the temperature in the thermostat within the normal working range of the electronic devices. (5) A user interface running in the computer, which is mainly used for parameter setting, sending of control commands, receiving equipment status information, and displaying the acquired status information and imaging results. (6) An ice core slice bracket for supporting the ice core slice and a tool body for carrying all equipments.

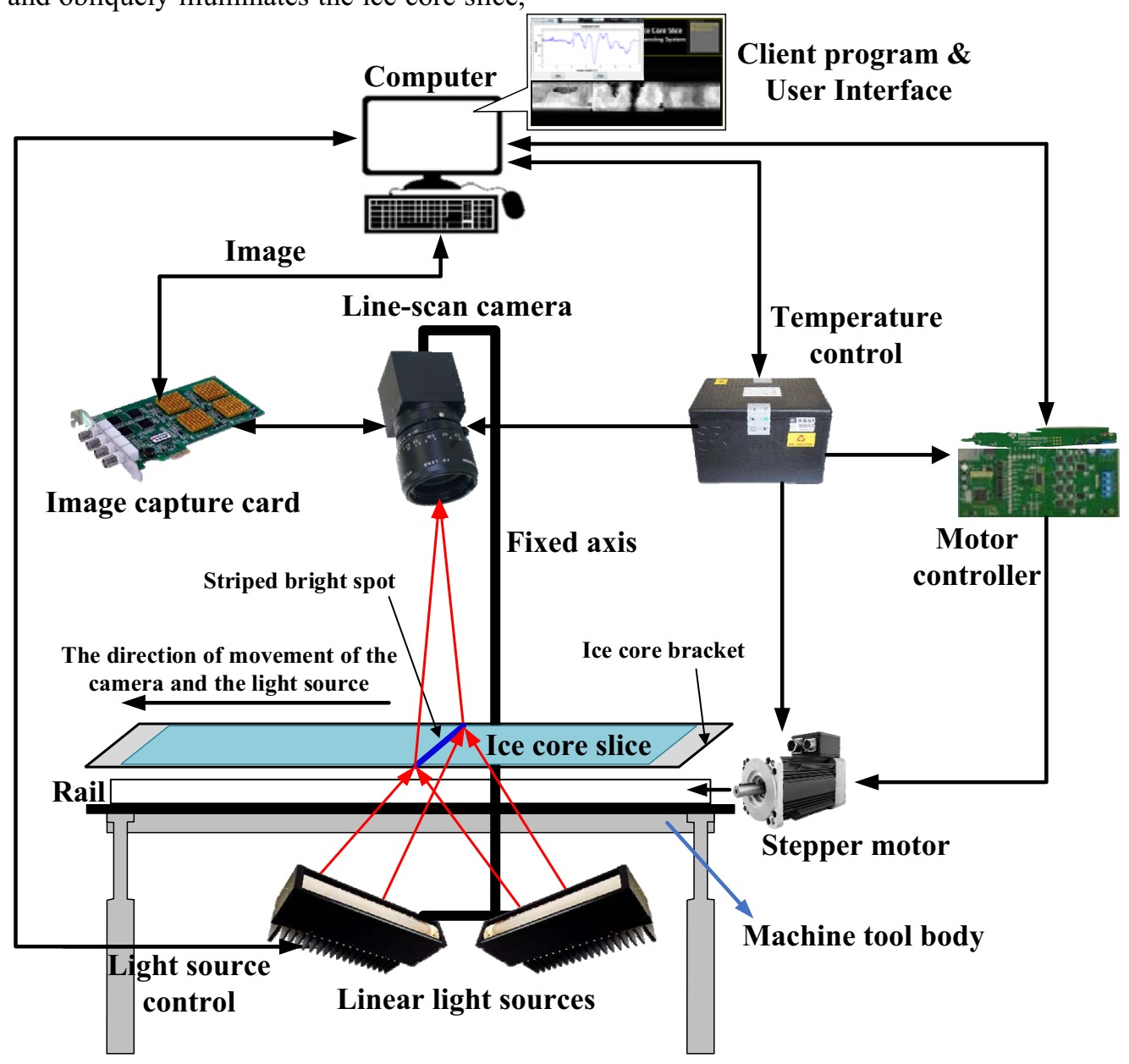

Figure 1. The overall structure diagram of the proposed tool

\section{Implementation of the proposed ice core slice scanning tool}

In Figure 1, according to the design requirements, the linescan camera uses the Mars Gigabit Ethernet line-scan industrial camera of Vision Datum, which can achieve $501 \mathrm{p} / \mathrm{mm}$ (10MP) resolution with a special lens. The image capture card uses the GigE interface capture card that is compatible with the camera from Vision Datum. The linear light source adopts commercial adjustable linear LED light source with Light Shaping Diffuser (LSD) film. The diffusion effect of the LSD film makes the light 
emitted by the LED light source more uniform. The remaining major equipments, including motor and motor controller, as well as special man-machine interface running in the computers, will be discussed in detail below.

\subsection{Motor and its controller}

Aiming at the low temperature, low speed and highprecision constant speed control requirements of the motor, we select the planetary reduction stepper motor 57PG2400-020 of Samsr 57PG series.
In order to ensure the stability and efficiency of the ice core slice scanning, the stepper motor controller must drive the motor to control the movement of the guide rail more precisely. TI's high-precision subdivision stepper motor control chip DRV8711 is adopted to control the stepper motor 57PG2400-020. DRV8711 has a built-in 256 subdivision indexer, which can realize smaller subdivision of the step angle of the stepper motor. The structure of the stepper motor drive control circuit based on DRV8711 used in this study is shown in Figure 2(a), and the actual circuit is shown in Figure 2(b). Among them, MCU selects STM32F103 of STMicroelectronics.

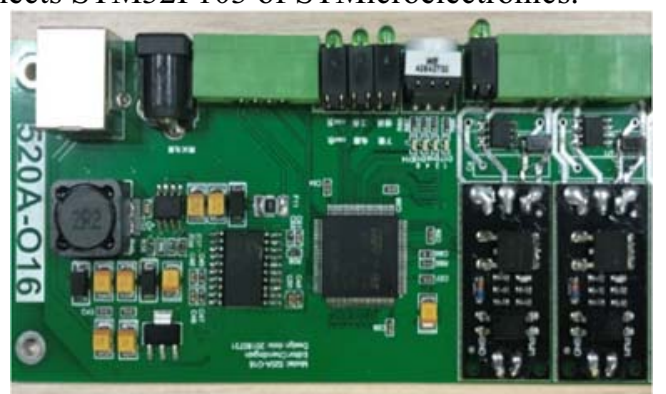

(b)

(a)

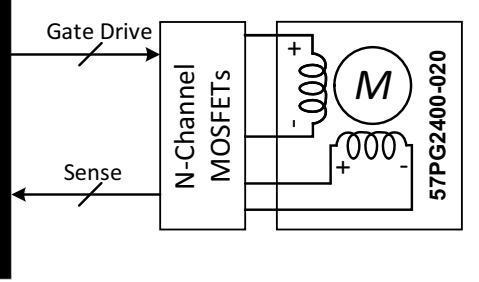

Figure 2. The proposed stepper motor controller based on DRV8711.

Since the stepping angle of the stepper motor 57PG2400-020 is 0.09 degrees, if the subdivision is set to 256 , each pulse of the main control chip can control the stepper motor to rotate only 0.0003515625 degrees, thus realizing the fine control of the stepper motor movement.

In addition, the MCU configures DRV8711 through the SPI communication interface, including enabling the stepper motor, setting the number of subdivision steps and dead time, etc. After the configuration of the stepper motor is completed, the MCU can use DIR pin to control the rotation direction of the stepper motor. The MCU controls the speed of the stepper motor by inputting Pulse Width Modulation (PWM) pulses to the STEP pin. The greater the frequency of the pulse, the faster the stepper motor rotates, and the longer the rail moves in a certain period of time.

\subsection{Client programs and user interface}

The client program of the system mainly has three parts, namely equipment control, data acquisition and data processing, as shown in Figure 3. The control part is mainly used to perform the control of the motor controller and then the motor, the setting of the camera, the adjustment and control of the brightness of the lighting system, and the temperature control. The data acquisition part is mainly used to complete the collection and display of the ice core image and the temperature. The data processing part performs corresponding data processing on the collected data, such as the grayscale analysis on the ice core image.

The client program is developed by Visual Studio 2013 platform. It sends commands to the motor controller, linescan camera, light sources and temperature controller through the RS485/RS232/GigE bus to complete their configuration or control. Meanwhile, it receives the collected ice core slice image and the temperature value in the thermostat through the RS485/GigE bus and displays them on the graphical interface.

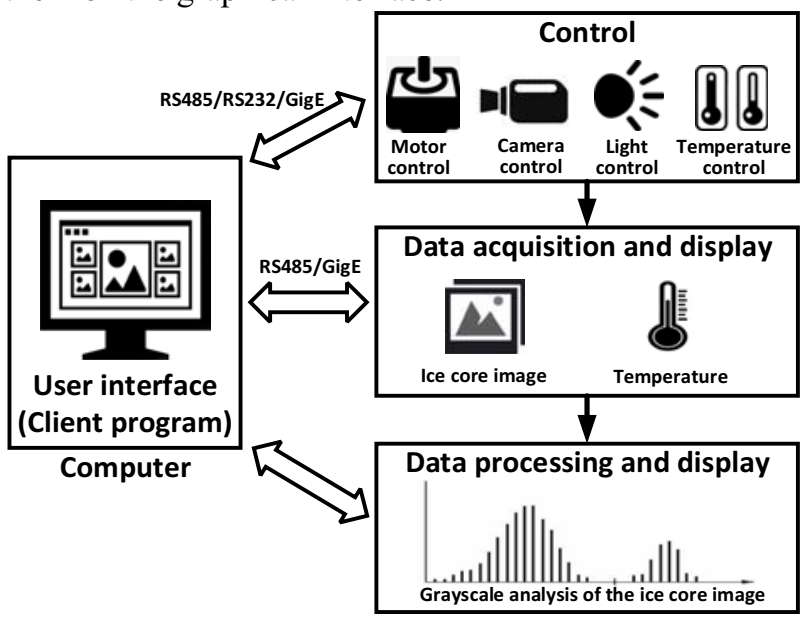

Figure 3. The diagram of the proposed client program.

The design of the user interface is based on the principle of simplicity and ease of use. It uses different areas in the interface to divide functions. The interface is mainly divided into four areas, namely the configuration and control area, the operation record area, the ice core image display area, and the grayscale analysis area (newly pop-up dialog box), as shown in Figure 4. The configuration and control area is mainly responsible for the parameter setting of the camera, light source and temperature control system, the movement direction and speed control of the motor, and the brightness adjustment of the light source. The operation record area can clearly display each step of the user's operation, accompanied by time tagging. The ice core image display area is used to display the scanned image of the ice core slice. The grayscale analysis interface will pop up as the user clicks 
the "Generate Grayscale Curve" button, and displays the grayscale statistical curve of the current ice core slice

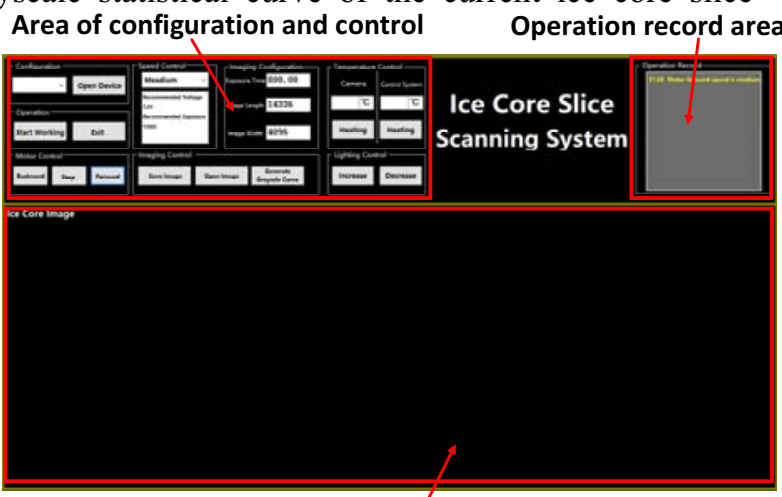

Display area of ice core image scanned image.

\section{Area of grayscale analysis (newly pop-up)}

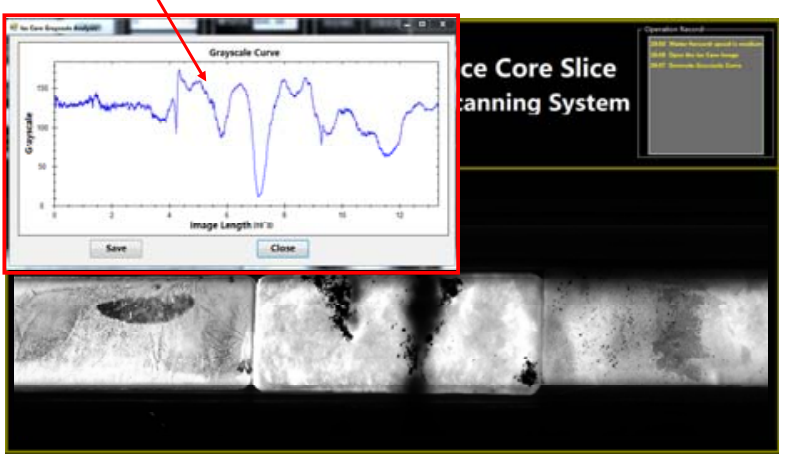

Figure 4. The example of the user interface.

\section{Experimental results}

\subsection{Scanning imaging test}

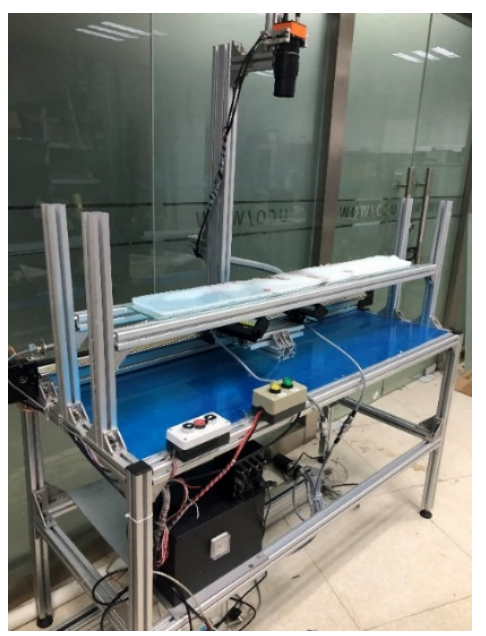

Figure 5. Photographs of the assembled tool.

During the test, we used some moulds to make a number of simulated ice core slices with a length of $40 \mathrm{~cm}$, a width of $12 \mathrm{~cm}$, and a thickness of $2 \mathrm{~cm}$ through a highlow temperature test chamber. Meanwhile, in order to simulate the impurities in the ice core, we mixed different impurities into the simulated ice core slices, including

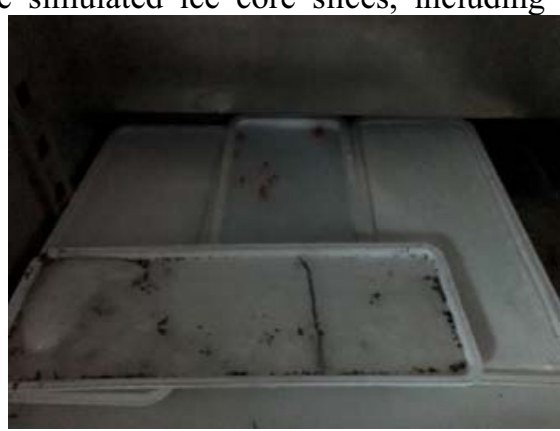

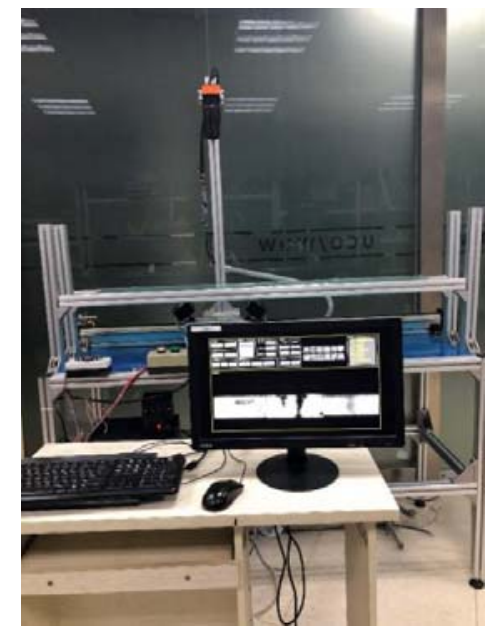

After the tests of all modules of the tool are completed, we assemble all the modules as a whole to perform the ice core slice scanning imaging test. The assembled ice core slice scanning tool is shown in Figure 5.

washing powder, soil, and Sprite. The simulated ice core slices used in the scanning imaging test are shown in Figure 6. The imaging results of multiple simulated ice core slices are shown in Figure 7. Scanning imaging results show that the proposed ice core slice scanning tool meets the design requirements.

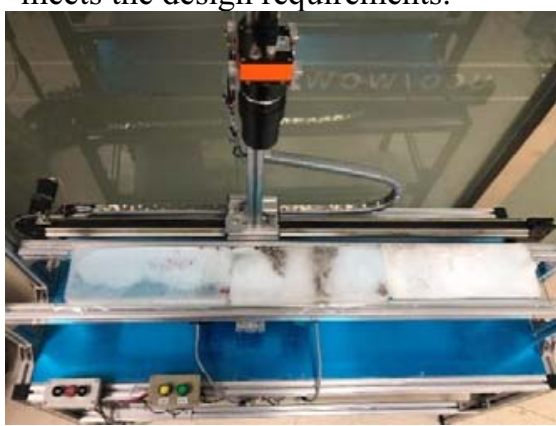

Figure 6. The simulated ice core slices used in the scanning imaging test. 

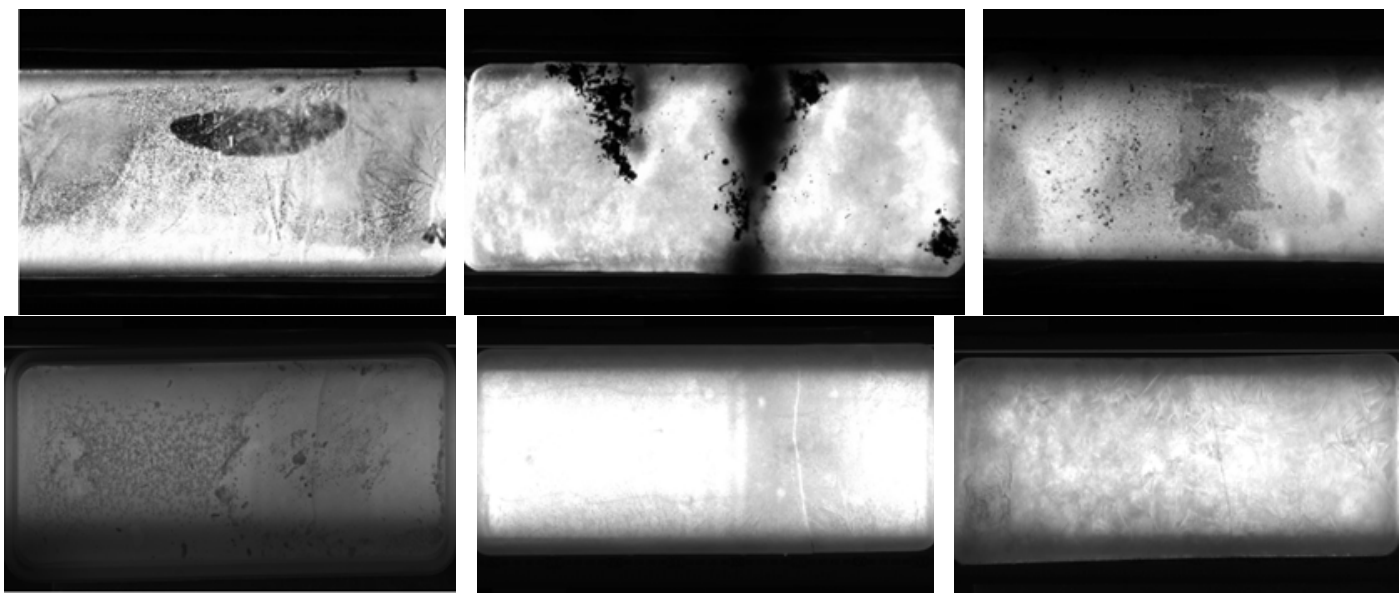

Figure 7. The imaging results of multiple simulated ice core slices.

\subsection{Comparison with the area-scan camera}

In this section, we compare the imaging results of the ice core slice based on the proposed line-scan camera with the imaging results of the area-scan camera based on image stitching. The comparison is mainly focused on the image quality.

Because the area-scan camera imaging method based on image stitching has higher requirements for illumination, the layout of the illumination system is more complicated than the proposed line-scan camera-based method, so that it is very easy to cause reflected light imaging and result in a decrease in imaging quality, as shown in Figure 8.

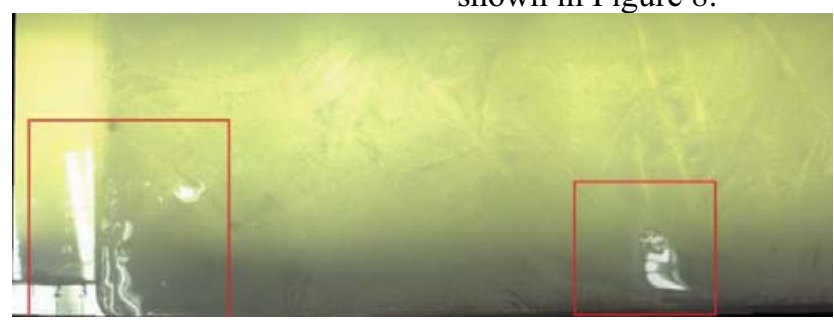

Figure 8. The example results of the area-scan camera imaging based on image stitching. The two red frames are the imaging result of the reflected light.

At the same time, as the pixels of the area-scan camera are distributed in a plane, the pixel value in a certain direction of the image will be limited by the aspect ratio, resulting in the resolution of the image being much lower than that of the line-scan camera. This makes the details of the captured ice core slice not clear enough, as shown in Figure 9. Figure 9(a) is the imaging results of an areascan camera based on image stitching. The tiny structures in the ice core slice are very blurred. Figure 9(b) are the imaging results of the same ice core slice by the proposed tool. All the tiny structures of the ice core slice are clearly visible in the figure.
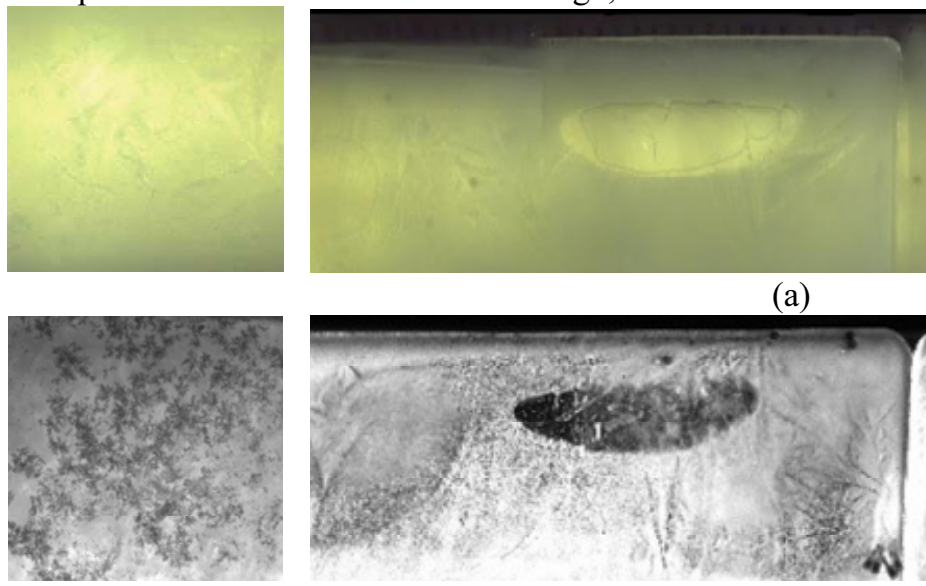

(b)

Figure 9. Comparison of the imaging results for the same simulated ice core slices. (a) are the imaging results of an area-scan camera based on image stitching. (b) are the imaging results of the proposed tool. 


\section{Conclusion}

In order to achieve high-resolution imaging of ice core slices, an ice core slice scanning tool based on line-scan camera is proposed in this study. The tool uses the highresolution characteristics of the line-scan camera to complete high-resolution imaging of the ice core slices through linkage with the linear light source. For the imaging requirements of line-scan cameras with long exposure time and fixed light sources, a motor drive and control system is developed to complete the uniform scanning control of the line-scan camera and light sources. A special control program and human-computer interaction interface are developed to realize the parameter setting, motor control and imaging result display. The test results show that the proposed ice core slice scanning tool meets the design requirements and its imaging results are significantly better than area-scan cameras under the same conditions.

\section{Acknowledgments}

This work was supported in part by the National Key Research and Development Project of China (Grants No. 2016YFC1400302).

We sincerely thank Prof. Sepp Kipfstuhl for his suggestions.

\section{References}

1. Brook E J \& Buizert C. (2018) Antarctic and global climate history viewed from ice cores. Nature, 558(7709): 200-208.

2. Mcgwire K C, Hargreaves G M, Alley R B, et al. (2008) An integrated system for optical imaging of ice cores. Cold Regions Science and Technology, 53: 216-228.

3. Takata M, Shoji H, Miyamoto A, et al. (2003) Comparative studies in method for stratigraphical structure measurement of ice cores: Identification of cloudy bands. Chinese Journal of Polar Science, 14: 73-80.

4. Sjgren B, Brandt O, Nuth C, et al. (2014) Instruments and Methods Determination of firn density in ice cores using image analysis. Journal of Glaciology, 53: 413-419.

5. Kinnard C, Koerner R M, Zdanowicz C M, et al. (2008) Stratigraphic analysis of an ice core from the Prince of Wales Icefield, Ellesmere Island, Arctic Canada, using digital image analysis: High-resolution density, past summer warmth reconstruction, and melt effect on ice core solid conductivity. Journal of Geophysical Research, 113: D24120.

6. Krischke A, Oechsner U, Kipfstuhl S. (2015) Rapid Microstructure Analysis of Polar Ice Cores. Optik \& Photonik, 10: 32-35.

7. Weikusat I, Jansen D, Binder T, et al. (2017) Physical analysis of an Antarctic ice core-towards an integration of micro-and macrodynamics of polar ice.
Philosophical Transactions of the Royal Society A: Mathematical, Physical and Engineering Sciences, 375: 20150347.

8. Svensson Anders, Søren Wedel Nielsen, Sepp Kipfstuhl, et al. (2005) Visual stratigraphy of the North Greenland Ice Core Project (NorthGRIP) ice core during the last glacial period. Journal of Geophysical Research Atmospheres, 110: D02108. 